\title{
A comprehensive model including preoperative peripheral blood inflammatory markers for prediction of the prognosis of diffuse spinal cord astrocytoma following surgery
}

\author{
Bo Pang ${ }^{1,2,4} \cdot$ Rui-Chao Chai ${ }^{1,2,3,4} \cdot$ Yao-Wu Zhang ${ }^{1,2} \cdot$ Yu-Zhou Chang ${ }^{1,2} \cdot$ Wei-Hao Liu ${ }^{1,2} \cdot$ Wen-Qing Jia ${ }^{1,2,3,4}$. \\ Yong-Zhi Wang ${ }^{1,2,3,4}$
}

Received: 26 June 2020 / Revised: 27 November 2020 / Accepted: 6 January 2021 / Published online: 25 January 2021

(C) The Author(s) 2021

\begin{abstract}
Purpose Due to the rarity of diffuse spinal cord astrocytoma, an effective model is still lacking to stratify their prognosis. Here, we aimed to establish a prognostic model through comprehensively evaluating clinicopathological features and preoperative peripheral blood inflammatory markers in 89 cases.

Methods We performed univariate and multivariate Cox regression to identify prognosis factors. The Kaplan-Meier curves and ROC curves were employed to compare the prognostic value of selected factors.

Results In addition to clinicopathological factors, we revealed the preoperative peripheral blood leukocyte count, neutrophilsto-lymphocytes ratio (NLR), and platelet-to-lymphocyte ratio (PLR) were also significantly correlated with overall survival of spinal cord astrocytoma in univariate Cox regression, and NLR was still significant in multivariate Cox analysis. Further, we demonstrated that NLR $\leq 3.65$ and preoperative McCormick score (MMS) $\leq 3$ were independently correlated with better survival of WHO grade IV tumors. Meanwhile, Ki-67 $<10 \%$ and resection extent $\geq 90 \%$ were independent prognostic factors in WHO grade II/III tumors. Finally, we developed a prognostic model that had better predictive efficiencies than WHO grade and histological grade for 1-year $(\mathrm{AUC}=76.6), 2$ - year $(\mathrm{AUC}=80.9)$, and 3-year $(\mathrm{AUC}=80.3)$ survival. This model could classify tumors into 4 classifications with increasingly poor prognosis: 1 , WHO grade II/III, with Ki-67 $<10 \%$ and resection extent $\geq 90 \% ; 2$, WHO grade II/III, Ki-67 $\geq 10 \%$ or resection $<90 \% ; 3$, WHO grade IV, NLR $\leq 3.65$ and MMS $\leq 3$; 4, WHO grade IV, with NRL $>3.65$ or MMS $=4$.

Conclusion We successfully constructed a comprehensive prognostic model including preoperative peripheral blood inflammatory markers, which can stratify diffuse spinal cord astrocytoma into 4 subgroups.
\end{abstract}

Keywords Spinal cord astrocytoma $\cdot$ Preoperative peripheral blood inflammatory markers $\cdot$ Prognostic model Neutrophils-to-lymphocytes ratio $\cdot$ McCormick score

Bo Pang and Rui-Chao Chai contributed equally to this work.

Supplementary Information The online version of this article (https://doi.org/10.1007/s00586-021-06724-4) contains

supplementary material, which is available to authorized users.

Rui-Chao Chai

chairuichao_glia@163.com

Wen-Qing Jia

coffeemd@163.com

Yong-Zhi Wang

yongzhiwang_bni@163.com

Extended author information available on the last page of the article

\begin{tabular}{ll}
\multicolumn{2}{l}{ Abbreviations } \\
WHO & World Health Organization \\
OS & Overall survival \\
NLR & Neutrophils-to-lymphocytes ratio \\
PLR & Platelet-to-lymphocyte ratio \\
MLR & Monocyte-to-lymphocyte ratio \\
RT & Radiation therapy \\
MMS & McCormick score \\
HR & Hazard ratio \\
MRI & Magnetic resonance images \\
FFPE & Formalin-fixed paraffin-embedde \\
PCR & Polymerase Chain Reaction
\end{tabular}

Abbreviations

OS Overall survival

NLR Neutrophils-to-lymphocytes ratio

PLR Platelet-to-lymphocyte ratio

MLR Monocyte-to-lymphocyte ratio

RT Radiation therapy

MMS McCormick score

HR Hazard ratio

MRI Magnetic resonance images

PCR Polymerase Chain Reaction 


\section{Introduction}

Diffuse spinal cord astrocytoma (WHO grade II-IV) is one of the most common types of intramedullary spinal cord malignant tumors [1-7]. Despite this, they are relatively rare compared to their intracranial counterparts $[5$, 6]. Besides, only a small amount of tissue could be available during the procedure of spinal cord surgery. Due to these reasons, current knowledge of diffuse spinal cord astrocytoma comes from only a handful of studies with a limited number of cases [7-9]. The classification of spinal cord astrocytoma is also largely referring to the standard of their intracranial counterparts [10]. However, it has been reported that the clinicopathological features were distinct between the spinal cord and brain tumors, and the prognosis of diffuse spinal cord astrocytoma is also largely varied within the same classification $[1,6,7,11-13]$. In general, it is difficult to accurately stratify the prognosis of spinal cord astrocytoma based on the current classification system.

Accurate prediction of spinal cord glioma prognosis is critical for postoperative management and treatment. Currently, the common treatment for spinal cord gliomas is maximal safe surgical resection, and adjuvant chemotherapy and radiation therapy (RT) are also used for recurrent or progressive residual tumors [13-15]. However, an aggressive and combined modality treatment approach may be adopted for a very poor prognosis patient, while avoidance of long-term nerve injury should be considered for patients with a relatively favorable prognosis $[7,16]$. Therefore, it is urgent to develop a model to accurately predict the survival of patients with diffuse spinal cord astrocytoma.

Although our current knowledge is incomplete for prognosis factors of spinal cord gliomas, some clinicopathological features had been reported to be associated with the prognosis of the spinal cord [4-7, 12]. Histologic grade, WHO grade, preoperative neurological status, surgical extent, and $\mathrm{Ki}-67$ index had been reported as prognostic factors in previous studies [3, 5, 14, 15, 17, 18]. However, these findings still should be verified in a relatively large cohort. In our recent study, we confirmed the prognostic value of both histologic grade and WHO grade in spinal cord astrocytoma, and we also revealed that TERT promoter mutation and $\mathrm{H} 3 \mathrm{~K} 27 \mathrm{M}$ mutation had prognostic value in WHO grade II/III and WHO grade IV tumors, respectively [1].

Currently, immunity and inflammatory response are critical for tumor initiation and progression [19-21]. Some preoperative peripheral blood inflammatory markers, such as neutrophil-to-lymphocyte ratio (NLR), plateletto-lymphocyte ratio (PLR), and monocyte-to-lymphocyte ratio (MLR), also have prognostic value in brain glioma [22-24]. It has been indicated that the immune microenvironment is different between brain and spinal cord gliomas [25]. Thus, in addition to the traditional clinicopathological factors, it is necessary to clarify whether peripheral blood markers also have prognostic value in spinal cord gliomas.

Here, we systemically analyzed the prognostic factors, including peripheral blood inflammatory markers, in 89 cases of spinal cord astrocytoma. We aimed to (1) investigate the prognostic value of peripheral blood markers in spinal cord gliomas; (2) construct a comprehensive prognostic model with clinicopathological features and peripheral blood markers of spinal cord astrocytoma.

\section{Materials and methods}

\section{Samples collection}

In a previous study, we have systemically compared the molecular features of 83 diffuse spinal cord gliomas with or without H3 K27M mutation [1]. In this study, another 6 cases whose prognostic information was obtained recently were also included to develop a comprehensive prognostic model for patients with diffuse spinal cord gliomas.

\section{Clinical and pathological features of samples}

The surgery resection extent was estimated using postsurgery magnetic resonance images (MRI). Based on the MRI results, the surgery resection extent was defined as gross total resection ( $\geq 90 \%)$, subtotal resection $(\geq 50 \%$ and $<90 \%)$, and open biopsy $(<50 \%)[1,26]$. The surgery resection extent was independently determined by two experienced radiologists, who were blinded to the clinical data of the patients. In case of a discrepancy, the two observers simultaneously reviewed the images to achieve a consensus. The MMS was calculated according to the standard previously reported [27].

The histological pathological and molecular features were obtained as previously reported [1]. The histopathological grade was determined by the routine evaluation of FFPE samples with hematoxylin and eosin (H\&E) staining based on 2016 WHO classification. The H3 K27M-mutant status (ABE419; Millipore, Billerica, MA, USA; 1:800) and Ki-67 expression status (MIB-1; Labvision, Fremont, CA, USA; 1:50) were determined by immunohistochemistry. The TERT promoter $\mathrm{C} 228 \mathrm{~T}$ and $\mathrm{C} 250 \mathrm{~T}$ mutations were determined by the pyrosequencing after PCR amplification.

In addition to the clinical and pathological characteristics, we also collected the preoperative peripheral blood cell data of these patients from the electronic medical record system, 
including the leukocyte count, the proportion of five leukocyte subtypes (neutrophil, lymphocyte, monocyte, eosinophil, and basophil). We also calculated the NLR, PLR, and MLR for each patient. These patients did not catch acute conditions like bacterial or viral infections or drug treatments that might affect the human body's immune system.

The demographic and clinical characteristics of all cases used in this study were summarized in Table 1. All the patient's consent has been obtained during their hospitalization. The procedure of this study has been approved by the Institutional Review Board and Ethics Committee of Beijing Tiantan Hospital (Beijing, China).

\section{Statistical analysis}

One-way ANOVA and unpaired t-test were used to compare the distribution of markers in different groups. Overall survival (OS) was defined as the time interval from diagnosis to death. Univariate and multivariate Cox regression analyses were used to study the influence of different factors on OS. The optimal cut-off values of NLR, PLR, MLR, and other prognostic factors were computed by X-tile software [28]. The Kaplan-Meier method with log-rank testing was used to compare the OS of cases in different groups. $P$-value $<0.05$ was defined as significant. All of the statistics were performed by GraphPad Prism version 7.0 statistical software (GraphPad Software, La Jolla, CA, USA) or IBM SPSS Statistics 22 (IBM, Armonk, NY, USA).

\section{Results}

\section{Clinical characteristics of patients}

A total of 89 patients with diffuse spinal cord astrocytoma were included in this study, including 54 male patients $(60.70 \%)$ and 35 female patients (39.30). The detailed information on the clinicopathological features and preoperative peripheral makers of all patients were summarized in Table 1. The age of all patients ranged from 6 to 63, with a median and an average age of 31 and 32.37, respectively. The preoperative McCormick score (MMS) of all patients were distributed as follows: 3 patients $(3.40 \%)$ with $\mathrm{MMS}=1$; 36 patients $(40.40 \%)$ with $\mathrm{MMS}=2 ; 34$ patients $(38.20 \%)$ with $\mathrm{MMS}=3$, and 16 patients $(18.00 \%)$ with $\mathrm{MMS}=4$. In 46 patients $(51,70 \%)$, tumor resection was more than $90 \%$, in 14 patients $(15.70 \%)$, tumor resection was between 50 and $90 \%$, and in 29 patients (32.60), tumor resection was less than $50 \%$. According to our follow-up information, 57 patients received postoperative radiotherapy and 25 patients received chemotherapy.

For the pathological characteristics, there were 56 histological grade II tumors $(62.90 \%), 20$ histological grade
Table 1 Clinical characteristics of patients in this study

\begin{tabular}{|c|c|c|}
\hline Characteristics & Number & Mean \pm SD or percentage \\
\hline \multicolumn{3}{|l|}{ Clinical factors } \\
\hline \multicolumn{3}{|l|}{ Gender } \\
\hline Male & 54 & $60.70 \%$ \\
\hline Female & 35 & $39.30 \%$ \\
\hline \multicolumn{3}{|l|}{ Age } \\
\hline Mean & 32.37 & $32.37 \pm 14.155$ \\
\hline Median (Range) & 31 & $31.5(6-63)$ \\
\hline \multicolumn{3}{|l|}{ McCormick score (MMS) } \\
\hline 1 & 3 & $3.40 \%$ \\
\hline 2 & 36 & $40.40 \%$ \\
\hline 3 & 34 & $38.20 \%$ \\
\hline 4 & 16 & $18.00 \%$ \\
\hline \multicolumn{3}{|l|}{ Surgery resection extent } \\
\hline Resection $\geq 90 \%$ & 46 & $51.70 \%$ \\
\hline $50 \% \leq$ Resection $<90 \%$ & 14 & $15.70 \%$ \\
\hline Resection $<50 \%$ & 29 & $32.60 \%$ \\
\hline \multicolumn{3}{|l|}{ Chemotherapy } \\
\hline Yes & 25 & $28.10 \%$ \\
\hline No & 52 & $58.40 \%$ \\
\hline NA & 12 & $13.50 \%$ \\
\hline \multicolumn{3}{|l|}{ Radiotherapy } \\
\hline Yes & 57 & $64.00 \%$ \\
\hline No & 20 & $22.50 \%$ \\
\hline NA & 12 & $13.50 \%$ \\
\hline \multicolumn{3}{|l|}{ Pathological factors } \\
\hline \multicolumn{3}{|l|}{ Ki-67 } \\
\hline $\mathrm{Ki}-67 \geq 10 \%$ & 46 & $51.70 \%$ \\
\hline Ki-67<10\% & 43 & $48.30 \%$ \\
\hline \multicolumn{3}{|l|}{ H3K27M mutation } \\
\hline Yes & 36 & $40.40 \%$ \\
\hline No & 53 & $59.60 \%$ \\
\hline \multicolumn{3}{|l|}{ TERT promoter mutation } \\
\hline Yes & 13 & $14.77 \%$ \\
\hline No & 45 & $51.14 \%$ \\
\hline NA & 31 & $35.23 \%$ \\
\hline \multicolumn{3}{|l|}{ WHO grade } \\
\hline II & 38 & $42.70 \%$ \\
\hline III & 9 & $10.10 \%$ \\
\hline IV & 42 & $47.20 \%$ \\
\hline \multicolumn{3}{|l|}{ Histological grade } \\
\hline Grade II & 56 & $62.90 \%$ \\
\hline Grade III & 20 & $22.47 \%$ \\
\hline Grade IV & 13 & $14.60 \%$ \\
\hline \multicolumn{3}{|c|}{ Hematological marker factors } \\
\hline Leukocyte count & 88 & $6.922 \pm 2.494$ \\
\hline NEUT\% & 88 & $60.647 \pm 10.428$ \\
\hline LY\% & 88 & $31.185 \pm 9.425$ \\
\hline MONO\% & 88 & $6.115 \pm 1.817$ \\
\hline $\mathrm{EO} \%$ & 88 & $1.686 \pm 1.578$ \\
\hline BA $\%$ & 88 & $0.367 \pm 0.286$ \\
\hline
\end{tabular}


Table 1 (continued)

\begin{tabular}{lll}
\hline Characteristics & Number & Mean \pm SD or percentage \\
\hline NLR & 88 & $2.722 \pm 3.741$ \\
PLR & 88 & $137.840 \pm 81.389$ \\
MLR & 88 & $0.222 \pm 0.098$ \\
\hline
\end{tabular}

III tumors (22.47\%), and 13 histological grade IV patients $(14.60 \%)$. Among these patients, 36 patients $(40.40 \%)$ with H3 K27M mutation, and 46 patients $(51.70 \%)$ showed Ki-67-positive nuclei $\geq 10 \%$. There were 13 cases with TERT promoter mutation in 58 cases had TERT promotermutant information. Based on the World Health Organization (WHO) 2016 classification of tumors of the central nervous system, there were $38 \mathrm{WHO}$ grade II tumors $(42.70 \%), 9$ grade III tumors $(10.10 \%)$, and 42 grade IV tumors $(47.20 \%)$ which included 30 histological grade II/ III tumors with $\mathrm{H} 3 \mathrm{~K} 27 \mathrm{M}$ mutation.

We also collected the information on preoperative peripheral makers of 88 cases. The mean $\pm S D$ value for leukocyte was $6.922 \pm 2.494$, and the average proportions for neutrophil, lymphocyte, monocyte, eosinophil, and basophil were $60.65 \%, 31.19 \%, 6.12 \%, 1.69 \%$, and $0.37 \%$, respectively. The mean values of NLR, PLR, and MLR were $2.72,137.84$, and 0.22 , respectively.

\section{Preoperative peripheral blood inflammatory markers could serve as prognostic indicators in all diffuse spinal cord astrocytoma}

Initially, we systemically analyzed the prognostic value of each factor in all diffuse astrocytic tumors. In addition to clinicopathological factors, such as age $(P=0.08$, the hazard ratio $(\mathrm{HR}=1.029)$, MMS $(P<0.0001, \mathrm{HR}=1.73)$, surgery resection extent $(P=0.005, \mathrm{HR}=0.377)$, WHO grade $(P<0.0001, \mathrm{HR}=3.062)$, and Ki-67 $(P<0.0001$, $\mathrm{HR}=0.173)$, the preoperative peripheral blood inflammatory markers included leukocyte count $(P=0.023, \mathrm{HR}=1.135)$, neutrophils-to-lymphocytes ratio (NLR, $P<0.0001$, $\mathrm{HR}=1.237$ ), and platelet-to-lymphocyte ratio (PLR, $P<0.0001, \mathrm{HR}=1.007)$ were also significantly correlated with the overall survival (OS) of all spinal cord astrocytoma in the univariate Cox analysis (Table 2).

Then, we studied the OS-stratifying abilities of these preoperative peripheral blood inflammatory markers by Kaplan-Meier curves (Fig. 1a-c). The results indicated that the OS of patients could be significantly stratified by leukocyte counts (Fig. 1a, $P<0.0001$ ), NLR (Fig. 21B $P=0.0006$ ), and PLR (Fig. 1c, $P=0.0123$ ).

We also compared the values of leukocyte count, NLR, and PLR in cases with different WHO grades. The results indicated that there was no significant difference for these factors among cases with different WHO grades (Fig. 1d-f). To further study the prognostic independence of these
Table 2 Univariate and Multivariate Cox analysis in all diffused spinal cord gliomas (WHO grade II-IV)

\begin{tabular}{|c|c|c|c|c|}
\hline \multirow[t]{2}{*}{ Factors } & \multicolumn{2}{|c|}{ Univariate analysis } & \multicolumn{2}{|c|}{ Multivariate analysis } \\
\hline & $P$ value & HR $[95 \% \mathrm{CI}]$ & $P$ value & $\mathrm{HR}[95 \% \mathrm{CI}]$ \\
\hline \multicolumn{5}{|l|}{ Clinical factors } \\
\hline Gender & 0.156 & $1.543[0.847-2.811]$ & - & - \\
\hline Age & 0.008 & $1.029[1.007-1.051]$ & 0.579 & $1.005[0.980-1.031]$ \\
\hline McCormick score (MMS) & $<0.0001$ & $2.587[1.736-3.855]$ & 0.018 & 1.783 [1.102-2.885] \\
\hline Surgery resection extent & 0.005 & $0.377[0.190-0.747]$ & 0.071 & $0.471[0.222-0.997]$ \\
\hline Radiotherapy & 0.952 & $0.973[0.402-2.356]$ & - & - \\
\hline Chemotherapy & 0.109 & $0.524[0.238-1.155]$ & - & - \\
\hline \multicolumn{5}{|l|}{ Pathological factors } \\
\hline WHO grade & $<0.0001$ & $3.062[2.016-4.652]$ & 0.006 & 1.993 [1.216-3.267] \\
\hline Ki-67 & $<0.0001$ & $0.173[0.084-0.356]$ & 0.038 & $0.421[0.186-0.951]$ \\
\hline \multicolumn{5}{|l|}{ Hematological marker factors } \\
\hline Leukocyte count & 0.023 & $1.135[1.017-1.266]$ & 0.82 & $1.032[0.850-1.253]$ \\
\hline NEUT\% $\%$ & 0.291 & $1.014[0.988-1.040]$ & - & - \\
\hline LY\% & 0.232 & $0.982[0.954-1.011]$ & - & - \\
\hline MONO\% & 0.628 & $1.045[0.875-1.248]$ & - & - \\
\hline $\mathrm{EO} \%$ & 0.764 & $0.967[0.779-1.201]$ & - & - \\
\hline BA\% & 0.409 & $0.640[0.221-1.848]$ & - & - \\
\hline NLR & $<0.0001$ & 1.237 [1.104-1.385] & 0.005 & $1.170[1.050-1.304]$ \\
\hline PLR & $<0.0001$ & 1.007 [1.003-1.010] & 0.729 & 0.999 [0.991-1.007] \\
\hline MLR & 0.076 & $11.926[0.775-183.580]$ & - & - \\
\hline
\end{tabular}



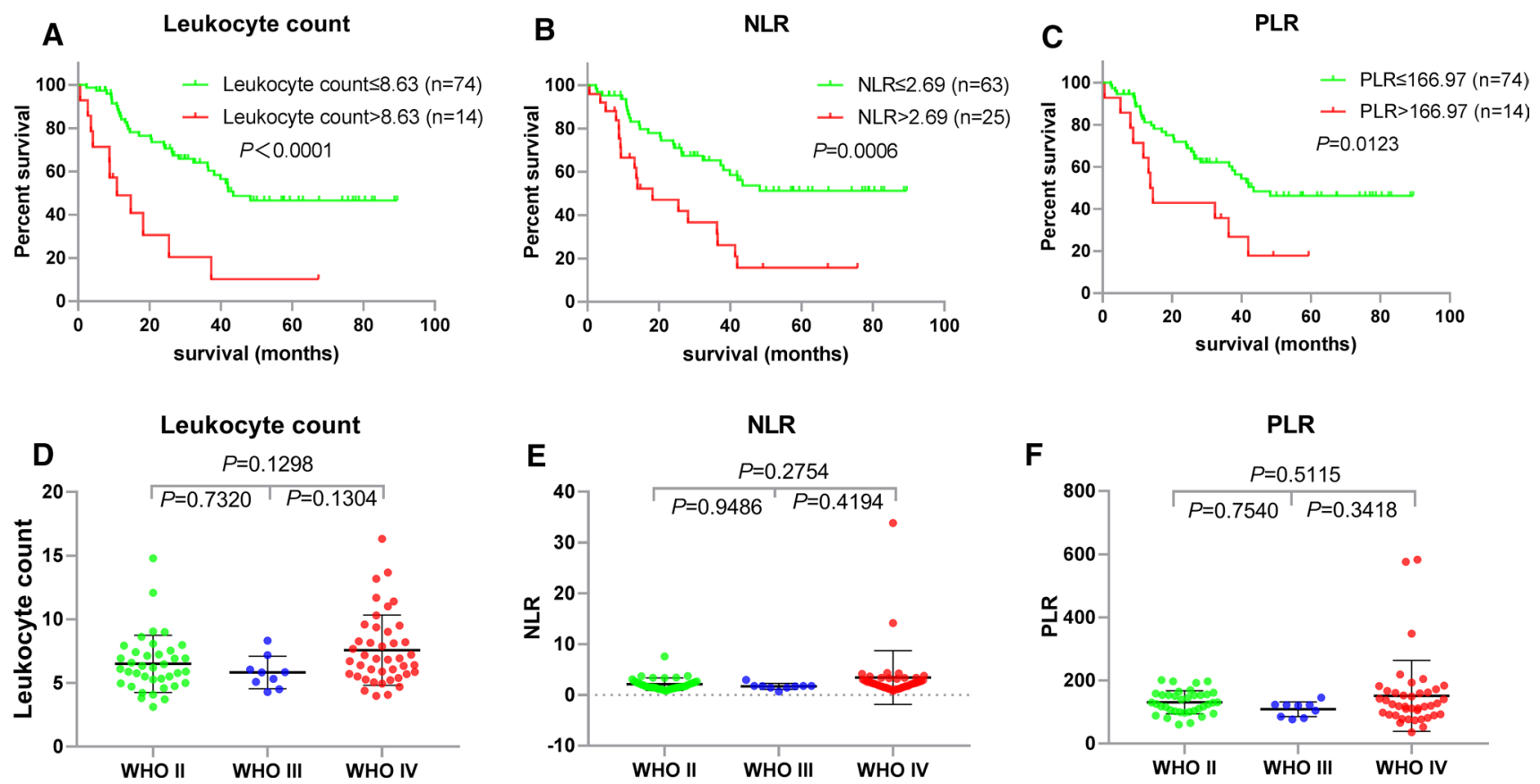

Fig. 1 The prognostic value and their distribution of preoperative peripheral blood inflammatory markers in diffuse spinal cord astrocytoma. a-c Kaplan-Meier survival curves for patients stratified by leu-

preoperative peripheral blood inflammatory markers, we performed multivariate Cox regression with all significant factors in univariate Cox analysis. The results indicated that MMS $(P=0.018$, HR $=1.783)$, WHO grade $(P=0.006$, HR $=1.993)$, Ki-67 index $(P=0.038, \mathrm{HR}=0.421)$, and NLR $(P=0.005, \mathrm{HR}=1.170)$ were still significantly correlated with OS of all diffuse spinal cord gliomas in multivariate Cox analysis, independent of age, surgery resection extent, leukocyte, and PLR (Table 2).

\section{Prognostic value of NLR and other clinicopathological features in non-WHO grade IV (WHO grade II/III) and WHO grade IV spinal cord gliomas}

Based on the integrated diagnosis of 2016 WHO classification, H3 K27M-mutant histological grade II/III spinal cord astrocytoma is also classified as WHO grade IV. The non-WHO grade IV (WHO grade II/III) tumors, which have similar molecular features, are commonly studied together as lower-grade gliomas [1, 9, 10, 29]. In addition to MMS, WHO grade, Ki-67 index, and NLR identified above, surgery resection extent and TERT promoter mutation are also potential prognostic factors in non-WHO grade IV tumors, while H3 K27M-mutant status and histological grade are also potential prognostic factor in WHO grade IV tumors kocyte count (a), NLR (b), and PLR (c). d-f The values of leukocyte count (d), NLR (e), and PLR (f) were compared among cases with different WHO grades

[1]. We also separately studied the prognostic factors in nonWHO grade IV and WHO grade IV tumors, respectively.

The result indicated that surgery resection extent, Ki-67 index, and TERT promoter mutation, rather than WHO grade, MMS, and NLR were significantly correlated with OS of patients with non-WHO grade IV tumors in univariate Cox analysis (Table 3 and Supplementary Fig. 1a, b). All of these three factors were still significantly correlated with OS in multivariate Cox analysis (Table 3 ). We also used these prognostic factors to further classify non-WHO grade IV spinal cord astrocytoma, and the result indicated that the classification model with Ki-67 index and resection extent (cutoff $\geq 90 \%$ ) could better stratify the nonWHO grade IV tumors (Supplementary Fig. $1 \mathrm{c}$ and d).

In WHO grade IV tumors, except for Ki-67, all other factors, including MMS, H3 K27M-mutant status, histological grade, and NLR, rather than Ki-67 significantly correlated to OS of patients in univariate Cox analysis (Table 4 and Supplementary Fig. 2a-e). In addition, MMS and NLR were significantly associated with OS in multivariate Cox analysis (Table 4). The WHO grade IV tumors could also be better stratified by a classification model derived from NRL and MMS (Supplementary Fig. 2f). We also observed the distribution of MMS, H3 K27M-mutant status, histological grade, NLR, and Ki-67 in WHO grade IV spinal cord astrocytoma (Supplementary Fig. 3). The result indicated that more than $50 \%$ of histological grade IV cases with $\mathrm{MMS}=4$, this may be the reason why $\mathrm{H} 3$ 

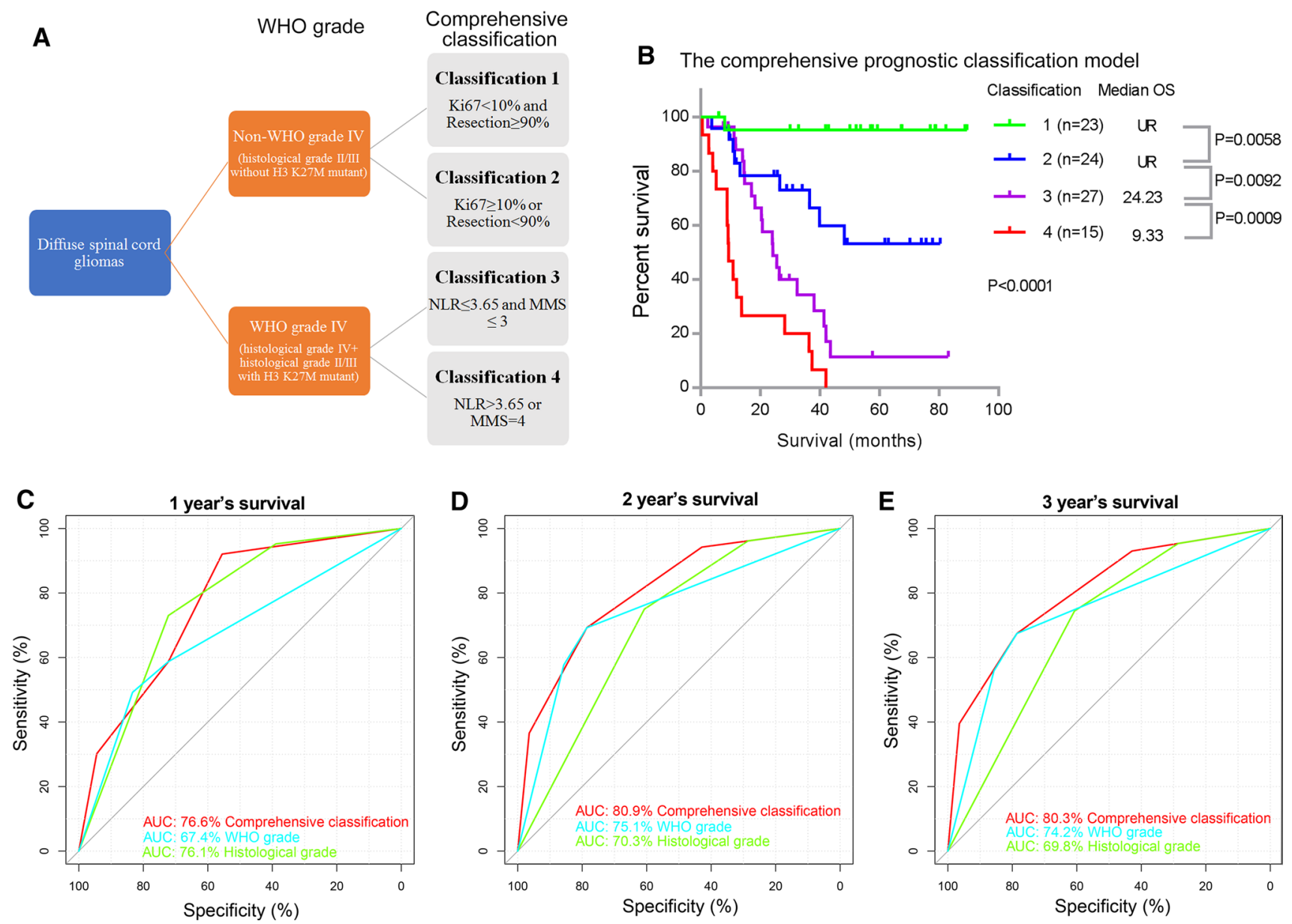

Fig. 2 The comprehensive prognostic classification model and it's prognostic value. a The diagrammatic graphic of the comprehensive prognostic classification model. b Kaplan-Meier survival curves for patients stratified by the comprehensive prognostic classification model. c-e ROC curves showed the predictive efficiencies of the comprehensive prognostic classification model, WHO grade, and histological grade on 1-year (c), 2-year (d), and 3-year (e) survival
Table 3 Univariate and Multivariate Cox analysis in lower grade spinal cord gliomas (WHO grade II-III)

Table 4 Univariate and Multivariate Cox analysis in WHO grade IV spinal cord gliomas

\begin{tabular}{|c|c|c|c|c|}
\hline \multirow[t]{2}{*}{ Factors } & \multicolumn{2}{|c|}{ Univariate analysis } & \multicolumn{2}{|c|}{ Multivariate analysis } \\
\hline & $P$ value & $\mathrm{HR}[95 \% \mathrm{CI}]$ & $P$ value & $\mathrm{HR}[95 \% \mathrm{CI}]$ \\
\hline WHO grade & 0.282 & $2.103[0.543-8.147]$ & - & - \\
\hline McCormick score & 0.692 & $1.202[0.484-2.988]$ & - & - \\
\hline Resection extent & 0.031 & $0.232[0.061-0.877]$ & 0.042 & $0.216[0.049-0.944]$ \\
\hline Ki-67 & 0.039 & $0.269[0.077-0.936]$ & 0.030 & $0.219[0.056-0.863]$ \\
\hline TERT mutation & 0.032 & $0.099[0.012-0.821]$ & 0.045 & $0.112[0.013-0.951]$ \\
\hline NLR & 0.677 & $1.096[0.712-1.689]$ & - & - \\
\hline
\end{tabular}

\begin{tabular}{|c|c|c|c|c|}
\hline \multirow[t]{2}{*}{ Factors } & \multicolumn{2}{|c|}{ Univariate analysis } & \multicolumn{2}{|c|}{ Multivariate analysis } \\
\hline & $P$ value & $\operatorname{HR}[95 \% \mathrm{CI}]$ & $P$ value & $\operatorname{HR}[95 \% \mathrm{CI}]$ \\
\hline McCormick score & 0.001 & 2.389 [1.396-4.090] & 0.005 & $2.278[1.290-4.021]$ \\
\hline $\mathrm{Ki}-67$ & 0.074 & 0.413 [0.157-1.089] & & \\
\hline H3K27M mutation & 0.009 & 3.785 [1.390-10.307] & 0.283 & $1.996[0.571-6.780]$ \\
\hline Histological grade & 0.023 & $1.636[1.080-2.769]$ & 0.284 & $1.334[0.781-2.322]$ \\
\hline NLR & 0.003 & $1.233[1.074-1.415]$ & 0.029 & $1.175[1.016-1.348]$ \\
\hline
\end{tabular}


K27M-mutant status and histological grade became nonsignificant in multivariate Cox analysis.

\section{Construction of a comprehensive prognostic model including preoperative peripheral blood inflammatory markers for all diffuse spinal cord gliomas}

Based on the above finding, we developed a comprehensive classification prognostic model. Firstly, we divided all diffuse spinal cord gliomas into non-WHO grade IV tumors and WHO grade IV tumors. Then, we could further stratify non-WHO grade IV by the surgery resection extent and Ki-67 index, and further stratified WHO grade IV tumors by the MMS and NLR (Fig. 2a). Finally, this model could stratify diffuse spinal cord gliomas into 4 classifications with increasingly poor prognosis: classification 1 , WHO grade II/III, with Ki-67 $<10 \%$ and resection extent $\geq 90 \%$; classification 2, WHO grade II/III, with Ki-67 $\geq 10 \%$ or resection $<90 \%$; classification 3 , WHO grade IV, with $\mathrm{NLR} \leq 3.65$ and MMS $\leq 3$; classification 4 , WHO grade IV, with NRL $>3.65$ or MMS $=4$ (Fig. 2a, b). In addition, we observed that this prognostic model had better prognostic efficiency than WHO grade and histological grade for 1-year (AUC $=76.6), 2$-year $(\mathrm{AUC}=80.9)$, and 3-year $(\mathrm{AUC}=80.3)$ survival (Fig. 2c-e).

\section{Discussion}

Accurate prognosis assessment is critical for the therapy design and clinical management of spinal cord astrocytoma $[9,16]$. However, accurate prognostic classification is still unmet medical demand for spinal cord astrocytoma. In this study, we systemically revealed the prognostic values of clinical features, pathological features, and preoperative peripheral blood inflammatory markers in 89 cases of diffuse spinal cord astrocytoma. Based on the identified prognostic factors, we successfully constructed a prognostic model, including WHO grade, surgery resection extent, Ki-67 index, preoperative MMS, and preoperative peripheral blood NLR. This model could stratify spinal cord gliomas into 4 classifications: classification 1, WHO grade II/III, with Ki-67 < 10\% and resection extent $\geq 90 \%$; classification 2 , WHO grade II/ III, with Ki- $67 \geq 10 \%$ or resection $<90 \%$; classification 3, WHO grade IV, with NLR $\leq 3.65$ and MMS $\leq 3$; classification 4 , WHO grade IV, with NRL $>3.65$ or $\mathrm{MMS}=4$, and patients had increasing poorer survival from classification 1 to classification 4 . This prognostic model had better predictive efficiency than WHO grade and histological grade for 1-year, 2-year, and 3-year survival.

Nowadays, it has been accepted that immunity and inflammation play important roles in the initiation and progression of tumors, such as pancreatic cancer, hepatocellular carcinoma, brain glioma, and other tumors [20, 21, 30, 31]. Systemic inflammation is associated with alterations in peripheral blood leukocytes that can be captured by the NLR [32]. The number of preoperative peripheral blood inflammatory cells, such as neutrophils, lymphocytes, monocytes, and their ratios, have been reported to be associated with the prognosis and immunotherapy response of cancers [22, 23]. In tumors of the central nervous system, a low NLR is associated with a better prognosis of brain gliomas has been demonstrated in several different studies [21, 24, 33]. In addition, NLR combined with other preoperative blood inflammatory markers could be used to differentially diagnose craniopharyngioma, pituitary tumor, and Rathke cleft cyst [19]. Here, our study first revealed that leukocyte count, NLR, and PLR also had prognostic value in diffuse spinal cord astrocytoma. We further demonstrated that NLR was an independent prognostic factor in WHO grade IV spinal cord gliomas, but not in spinal cord gliomas with WHO grade II/ III. This may be associated with the different growth features between non-WHO grade IV and WHO grade IV spinal cord astrocytoma, and non-WHO grade IV tumors have fewer infiltrates than WHO grade IV tumors [18]. This finding also suggested that the response of spinal cord astrocytoma to immunotherapy may be also different between WHO grade II/III tumors and WHO grade IV tumors.

Before the 2016 WHO classification of tumors of the central nervous system, it had been accepted that gross total resection was associated with better survival of histological grade II spinal cord astrocytoma [7, 9, 17]. However, the prognostic value of surgery resection extent in high grade (histological III and IV) spinal cord astrocytoma is still confused, and it seems that surgery resection only has prognostic value in anaplastic spinal cord astrocytoma (histological grade III), but not spinal cord glioblastoma (histological grade IV) [3, 15, 18]. Based on the integrated diagnosis of 2016 WHO classification, H3 K27M-mutant histological grade II/III spinal cord astrocytoma is also classified as WHO grade IV [10, 34], and WHO grade II/III tumors with similar molecular features are commonly studied together as lower-grade gliomas. Here, we demonstrated that resection extent $\geq 90 \%$ were significantly correlated with longer survival of patients with WHO grade II/III spinal cord gliomas, but not in WHO grade IV tumors. Interestingly, the preoperative MMS was significantly correlated with the survival of patients with WHO grade IV tumors but not patients with WHO grade II/III spinal cord astrocytoma. These findings provided a direct evidence for clinical management the spinal cord astrocytoma after the 2016 WHO classification of tumors of the central nervous system.

Molecular pathological features are also critical for predicting the prognosis of spinal cord astrocytoma. A high $\mathrm{Ki}-67$ index has been reported to be associated with poorer 
survival of 26 spinal cord astrocytoma [18]. In a recent study, we have demonstrated that TERT promoter has prognostic value in WHO grade II/III spinal cord astrocytoma [1]. Here, we also observed that both $\mathrm{Ki}-67 \geq 10 \%$ and TERT promoter mutation were associated with poorer survival of WHO grade II/III spinal cord astrocytoma in the univariate Cox regression.

The limitations of this study include its retrospective design, both enrolled patients and their preoperative blood routine examinations were from a single-center, a limited number of patients due to the rarity of spinal cord astrocytoma. In addition, only 13 histological grade IV patients enrolled in our cohort, this may be present a possible bias for the analysis of these patients. Future studies with largescale cases are warranted to verify our prognostic model.

In summary, our study demonstrated the prognostic value of preoperative peripheral blood inflammatory markers in patients with diffuse spinal cord astrocytoma in a relatively large cohort. We further revealed that Ki-67 $<10 \%$ and resection extent $\geq 90 \%$ were independently correlated with better survival of patients with WHO grade II/III spinal cord astrocytoma. Meanwhile, NLR $\leq 3.65$ and MMS $\leq 3$ were independently correlated with better survival of patients with WHO grade IV tumors. Based on this, we constructed a comprehensive prognostic model for diffuse spinal cord astrocytoma, and this prognostic model could provide a more accurate prognostic prediction for patients, which will provide better evidence for the management of patients with spinal cord astrocytoma.

Author contributions Pang Bo and Rui-Chao Chai and wrote the manuscript. Rui-Chao Chai, Pang Bo, Yao-Wu Zhang, Yu-Zhou Chang, and Yong-Zhi Wang conducted the data analysis and pathological information collection. Yao-Wu Zhang and Wen-Hao Liu collected clinical information. Yong-Zhi Wang and Wen-Qing Jia supervised the work.

Funding This study was funded by the National Natural Science Foundation of China (81903078, 81773208, 81891004), the Capital Characteristic Clinical Application Research and Achievement Promotion Project of China (Z17110001017068), the National Key Research and Development Program of China (2018YFC0115604), and Strategic Priority Research Program of the Chinese Academy of Sciences (XDA16040000).

Availability of data and materials The datasets used and/or analyzed during the current study available from the corresponding author on reasonable request.

Code availability All of the statistics were performed by GraphPad Prism version 7.0 statistical software (GraphPad Software, La Jolla, CA, USA) or IBM SPSS Statistics 22 (IBM, Armonk, NY, USA).

\section{Compliance with ethical standards}

Conflict of interest The authors have no potential conflicts of interest to declare.
Open Access This article is licensed under a Creative Commons Attribution 4.0 International License, which permits use, sharing, adaptation, distribution and reproduction in any medium or format, as long as you give appropriate credit to the original author(s) and the source, provide a link to the Creative Commons licence, and indicate if changes were made. The images or other third party material in this article are included in the article's Creative Commons licence, unless indicated otherwise in a credit line to the material. If material is not included in the article's Creative Commons licence and your intended use is not permitted by statutory regulation or exceeds the permitted use, you will need to obtain permission directly from the copyright holder. To view a copy of this licence, visit http://creativecommons.org/licenses/by/4.0/.

\section{References}

1. Chai R-C, Zhang Y-W, Liu Y-Q, Chang Y-Z, Pang B, Jiang T, Jia W-Q, Wang Y-Z (2020) The molecular characteristics of spinal cord gliomas with or without H3 K27M mutation. Acta Neuropathol Commun 8(1):40. https://doi.org/10.1186/s40478-02000913-w

2. Hoffman LM, Veldhuijzen van Zanten SEM, Colditz N, Baugh J, Chaney B, Hoffmann M, Lane A, Fuller C, Miles L, Hawkins C, Bartels U, Bouffet E, Goldman S, Leary S, Foreman NK, Packer R, Warren KE, Broniscer A, Kieran MW, Minturn J, Comito M, Broxson E, Shih C-S, Khatua S, Chintagumpala M, Carret AS, Escorza NY, Hassall T, Ziegler DS, Gottardo N, Dholaria H, Doughman R, Benesch M, Drissi R, Nazarian J, Jabado N, Boddaert N, Varlet P, Giraud G, Castel D, Puget S, Jones C, Hulleman E, Modena P, Giagnacovo M, Antonelli M, Pietsch T, Gielen GH, Jones DTW, Sturm D, Pfister SM, Gerber NU, Grotzer MA, Pfaff E, von Bueren AO, Hargrave D, Solanki GA, Jadrijevic Cvrlje F, Kaspers GJL, Vandertop WP, Grill J, Bailey S, Biassoni V, Massimino M, Calmon R, Sanchez E, Bison B, Warmuth-Metz M, Leach J, Jones B, van Vuurden DG, Kramm CM, Fouladi M (2018) Clinical, radiologic, pathologic, and molecular characteristics of long-term survivors of diffuse intrinsic pontine glioma (DIPG): a collaborative report from the international and european society for pediatric oncology dipg registries. J Clin Oncol: Off J Am Soc Clin Oncol 36(19):1963-1972. https://doi.org/10.1200/ JCO.2017.75.9308

3. Houten JK, Cooper PR (2000) Spinal cord astrocytomas: presentation, management and outcome. J Neurooncol 47(3):219-224

4. Zadnik PL, Gokaslan ZL, Burger PC, Bettegowda C (2013) Spinal cord tumours: advances in genetics and their implications for treatment. Nat Rev Neurol 9(5):257-266. https://doi.org/10.1038/ nrneurol.2013.48

5. McGirt MJ, Goldstein IM, Chaichana KL, Tobias ME, Kothbauer KF, Jallo GI (2008) Extent of surgical resection of malignant astrocytomas of the spinal cord: outcome analysis of 35 patients. Neurosurgery. https://doi.org/10.1227/01.NEU.0000335070 .37943 .09

6. Alvi MA, Ida CM, Paolini MA, Kerezoudis P, Meyer J, Barr Fritcher EG, Goncalves S, Meyer FB, Bydon M, Raghunathan A (2019) Spinal cord high-grade infiltrating gliomas in adults: clinico-pathological and molecular evaluation. Mod Pathol: Off J United States Can Acad Pathol, Inc 32(9):1236-1243

7. Abd-El-Barr MM, Huang KT, Moses ZB, Iorgulescu JB, Chi JH (2018) Recent advances in intradural spinal tumors. Neurooncology 20(6):729-742. https://doi.org/10.1093/neuonc/nox230

8. Jiang T, Mao Y, Ma W, Mao Q, You Y, Yang X, Jiang C, Kang C, Li X, Chen L, Qiu X, Wang W, Li W, Yao Y, Li S, Li S, Wu A, Sai K, Bai H, Li G, Chen B, Yao K, Wei X, Liu X, Zhang Z, Dai Y, Lv S, Wang L, Lin Z, Dong J, Xu G, Ma X, Cai J, Zhang W, Wang H, Chen L, Zhang C, Yang P, Yan W, Liu Z, Hu H, Chen J, Liu 
Y, Yang Y, Wang Z, Wang Z, Wang Y, You G, Han L, Bao Z, Liu Y, Wang Y, Fan X, Liu S, Liu X, Wang Y, Wang Q (2016) CGCG clinical practice guidelines for the management of adult diffuse gliomas. Cancer Lett 375(2):263-273. https://doi.org/10.1016/j. canlet.2016.01.024

9. Chai R-C, Li Y-M, Zhang K-N, Chang Y-Z, Liu Y-Q, Zhao Z, Wang Z-L, Chang Y-H, Li G-Z, Wang K-Y, Wu F, Wang Y-Z (2019) RNA processing genes characterize RNA splicing and further stratify lower-grade glioma. JCI Insight. https://doi. org/10.1172/jci.insight.130591

10. Louis DN, Perry A, Reifenberger G, von Deimling A, FigarellaBranger D, Cavenee WK, Ohgaki H, Wiestler OD, Kleihues P, Ellison DW (2016) The 2016 World Health Organization classification of tumors of the central nervous system: a summary. Acta Neuropathol 131(6):803-820. https://doi.org/10.1007/s0040 1-016-1545-1

11. Banan R, Christians A, Bartels S, Lehmann U, Hartmann C (2017) Absence of MGMT promoter methylation in diffuse midline glioma, H3 K27M-mutant. Acta Neuropathol Commun 5(1):98. https ://doi.org/10.1186/s40478-017-0500-2

12. Wang L, Li Z, Zhang M, Piao Y, Chen L, Liang H, Wei Y, Hu Z, Zhao L, Teng L, Lu D (2018) H3 K27M-mutant diffuse midline gliomas in different anatomical locations. Hum Pathol 78:89-96. https://doi.org/10.1016/j.humpath.2018.04.015

13. Chai R-C, Zhang K-N, Chang Y-Z, Wu F, Liu Y-Q, Zhao Z, Wang K-Y, Chang Y-H, Jiang T, Wang Y-Z (2019) Systematically characterize the clinical and biological significances of $1 \mathrm{p} 19 \mathrm{q}$ genes in 1p/19q non-codeletion glioma. Carcinogenesis 40(10):1229-1239. https://doi.org/10.1093/carcin/bgz102

14. Choi SH, Yoon HI, Yi S, Park JW, Cho J, Shin DA, Ha Y, Kim D-S, Kim SH, Lee S-K, Chang JH, Suh C-O (2019) Treatment outcomes of radiotherapy for primary spinal cord glioma. Strahlentherapie und Onkologie : Organ der Deutschen Rontgengesellschaft [et al] 195(2):164-174. https://doi.org/10.1007/s0006 6-018-1366-3

15. Liu J, Zheng M, Yang W, Lo S-FL, Huang J (2018) Impact of surgery and radiation therapy on spinal high-grade gliomas: a population-based study. J Neurooncol 139(3):609-616. https:// doi.org/10.1007/s11060-018-2904-7

16. Bady P, Kurscheid S, Delorenzi M, Gorlia T, van den Bent MJ, Hoang-Xuan K, Vauléon É, Gijtenbeek A, Enting R, Thiessen B, Chinot O, Dhermain F, Brandes AA, Reijneveld JC, Marosi C, Taphoorn MJB, Wick W, von Deimling A, French P, Stupp R, Baumert BG, Hegi ME (2018) The DNA methylome of DDR genes and benefit from RT or TMZ in IDH mutant low-grade glioma treated in EORTC 22033. Acta Neuropathol 135(4):601-615. https://doi.org/10.1007/s00401-018-1810-6

17. Diaz-Aguilar D, ReFaey K, Clifton W, Durcanova B, Chen SG, Deen HG, Bydon M, Trifiletti DM, Pichelmann MA, QuiñonesHinojosa A (2019) Prognostic factors and survival in low grade gliomas of the spinal cord: a population-based analysis from 2006 to 2012. J Clin Neurosci: Off J Neurosurg Soc Australas 61:14-21. https://doi.org/10.1016/j.jocn.2018.11.025

18. Ryu SJ, Kim JY, Kim KH, Park JY, Kuh SU, Chin DK, Kim KS, Cho YE, Kim SH (2016) A retrospective observational study on the treatment outcomes of 26 patients with spinal cord astrocytoma including two cases of malignant transformation. Eur Spine J: Off Publ Eur Spine Soc, Eur Spin Deform Soc Eur Sect Cerv Spine Res Soc 25(12):4067-4079

19. Chen M, Zheng S-H, Yang M, Chen Z-H, Li S-T (2018) The diagnostic value of preoperative inflammatory markers in craniopharyngioma: a multicenter cohort study. J Neurooncol 138(1):113122. https://doi.org/10.1007/s11060-018-2776-x

20. Coussens LM, Werb Z (2002) Inflammation and cancer. Nature 420(6917):860-867
21. Zhang Z-Y, Zhan Y-B, Zhang F-J, Yu B, Ji Y-C, Zhou J-Q, Bai Y-H, Wang Y-M, Wang L, Jing Y, Duan W-C, Sun C, Sun T, Zhao H-B, Li K, Wang W-Q, Li R-Y, Sun H-W, Zhai G, Wang S-K, Wei X-T, Yang B, Yan D-M, Liu X-Z, Wang W-W (2019) Prognostic value of preoperative hematological markers combined with molecular pathology in patients with diffuse gliomas. Aging 11(16):6252-6272. https://doi.org/10.18632/aging.102186

22. Hsueh C, Tao L, Zhang M, Cao W, Gong H, Zhou J, Zhou L (2017) The prognostic value of preoperative neutrophils, platelets, lymphocytes, monocytes and calculated ratios in patients with laryngeal squamous cell cancer. Oncotarget 8(36):60514-60527. https://doi.org/10.18632/oncotarget.16234

23. Bilen MA, Martini DJ, Liu Y, Lewis C, Collins HH, Shabto JM, Akce M, Kissick HT, Carthon BC, Shaib WL, Alese OB, Pillai RN, Steuer CE, Wu CS, Lawson DH, Kudchadkar RR, El-Rayes BF, Master VA, Ramalingam SS, Owonikoko TK, Harvey RD (2019) The prognostic and predictive impact of inflammatory biomarkers in patients who have advanced-stage cancer treated with immunotherapy. Cancer 125(1):127-134. https://doi.org/10.1002/ cncr.31778

24. Wang Z-L, Zhang C-B, Liu Y-Q, Wang Z, Jiang T (2019) Peripheral blood test provides a practical method for glioma evaluation and prognosis prediction. CNS Neurosci Ther 25(8):876-883. https://doi.org/10.1111/cns.13120

25. Ellis JA, Castelli M, Assanah M, Bruce JN, Canoll P, Ogden AT (2015) Unique microenvironmental responses to PDGF stimulation in brain and spinal cord gliomas determine tumor phenotype. J Neurooncol 123(1):27-33. https://doi.org/10.1007/s1106 0-015-1769-2

26. Yi S, Choi S, Shin DA, Kim DS, Choi J, Ha Y, Kim KN, Suh C-O, Chang JH, Kim SH, Yoon DH (2019) Impact of H3.3 K27M mutation on prognosis and survival of grade IV spinal cord glioma on the basis of new 2016 World Health Organization classification of the central nervous system. Neurosurgery 84(5):1072-1081. https://doi.org/10.1093/neuros/nyy 150

27. Kim MS, Chung CK, Choe G, Kim IH, Kim HJ (2001) Intramedullary spinal cord astrocytoma in adults: postoperative outcome. J Neurooncol 52(1):85-94

28. Camp RL, Dolled-Filhart M, Rimm DL (2004) X-tile: a new bioinformatics tool for biomarker assessment and outcome-based cutpoint optimization. Clinical Cancer Res: Off J Am Assoc Cancer Res 10(21):7252-7259

29. Binder H, Willscher E, Loeffler-Wirth H, Hopp L, Jones DTW, Pfister SM, Kreuz M, Gramatzki D, Fortenbacher E, Hentschel B, Tatagiba M, Herrlinger U, Vatter H, Matschke J, Westphal M, Krex D, Schackert G, Tonn JC, Schlegel U, Steiger HJ, Wick W, Weber RG, Weller M, Loeffler M (2019) DNA methylation, transcriptome and genetic copy number signatures of diffuse cerebral WHO grade II/III gliomas resolve cancer heterogeneity and development. Acta Neuropathol Commun 7(1):59. https://doi. org/10.1186/s40478-019-0704-8

30. Habtezion A (2015) Inflammation in acute and chronic pancreatitis. Curr Opin Gastroenterol 31(5):395-399. https://doi. org/10.1097/MOG.0000000000000195

31. Michelson N, Rincon-Torroella J, Quiñones-Hinojosa A, Greenfield JP (2016) Exploring the role of inflammation in the malignant transformation of low-grade gliomas. J Neuroimmunol 297:132-140. https://doi.org/10.1016/j.jneuroim.2016.05.019

32. Capone M, Giannarelli D, Mallardo D, Madonna G, Festino L, Grimaldi AM, Vanella V, Simeone E, Paone M, Palmieri G, Cavalcanti E, Caracò C, Ascierto PA (2018) Baseline neutrophil-tolymphocyte ratio (NLR) and derived NLR could predict overall survival in patients with advanced melanoma treated with nivolumab. J Immunother Cancer 6(1):74. https://doi.org/10.1186/ s40425-018-0383-1 
33. Wang P-F, Song H-W, Cai H-Q, Kong L-W, Yao K, Jiang T, Li S-W, Yan C-X (2017) Preoperative inflammation markers and IDH mutation status predict glioblastoma patient survival. Oncotarget 8(30):50117-50123. https://doi.org/10.18632/oncotarget .15235

34. Louis DN, Giannini C, Capper D, Paulus W, Figarella-Branger D, Lopes MB, Batchelor TT, Cairncross JG, van den Bent M, Wick W, Wesseling P (2018) cIMPACT-NOW update 2: diagnostic clarifications for diffuse midline glioma, H3 K27M-mutant and diffuse astrocytoma/anaplastic astrocytoma IDH-mutant. Acta Neuropathol 135(4):639-642. https://doi.org/10.1007/s0040 1-018-1826-y

Publisher's Note Springer Nature remains neutral with regard to jurisdictional claims in published maps and institutional affiliations.

\section{Authors and Affiliations}

\section{Bo Pang ${ }^{1,2,4} \cdot$ Rui-Chao Chai ${ }^{1,2,3,4} \cdot$ Yao-Wu Zhang ${ }^{1,2} \cdot$ Yu-Zhou Chang ${ }^{1,2} \cdot$ Wei-Hao Liu ${ }^{1,2} \cdot$ Wen-Qing Jia ${ }^{1,2,3,4}$. Yong-Zhi Wang ${ }^{1,2,3,4}$}

1 Department of Molecular Neuropathology, Beijing Neurosurgical Institute, Beijing Tiantan Hospital, Capital Medical University, No. 119 South 4th Ring West Road, Fengtai District, Beijing 100070, People's Republic of China

2 Department of Neurosurgery, Beijing Tiantan Hospital, Capital Medical University, No. 119 South 4th Ring West Road, Fengtai District, Beijing 100070, People's Republic of China
3 China National Clinical Research Center for Neurological Diseases, Beijing, People's Republic of China

4 Chinese Glioma Genome Atlas Network (CGGA), Beijing, People's Republic of China 